\title{
Dividing attention between the color and the shape of objects
}

\author{
ANNE-MARIE BONNEL \\ Centre de Recherche en Psychologie Cognitive CNRS-CNRC, Marseille, France \\ and \\ WILLIAM PRINZMETAL \\ University of California, Berkeley, Califormia
}

\begin{abstract}
In many dual-task experiments, the priority observers give to each task is experimentally varied. Most experiments using this methodology have studied the effect of dividing attention between spatially distinct objects. We examined performance when attention had to be divided between stimulus attributes other than spatial location. In the first experiment, observers identified the color and the shape of a single letter. Accuracy was the same for single- and dual-task conditions, and a trialby-trial analysis revealed a strong positive correlation in the correct identification of the color and the shape. In the second experiment, color and shape judgments were separated in space, with opposite results: Dual-task performance was worse than single-task performance, and the trial-by-trial analysis indicated a strong negative correlation between tasks. The results indicated that often only one dimension was processed within a trial. The results support object and space models of attention.
\end{abstract}

The goal of the research reported here was to determine when attention to one attribute adversely affects the identification of another attribute. Specifically, we investigated divided attention between color and shape (1) when observers report the color and the shape of the same object, and (2) when observers report the color of one object and the shape of another object. To the extent that color and shape analyzers are independent, attention to one attribute should not affect identification performance on the other attribute. Alternatively, if we attend to objects, the identification of attributes will be positively correlated if the attributes are from the same object and negatively correlated if they are from different objects.

We investigated the question of feature independence using a dual-task procedure in which observers were instructed to report both the color and shape of either one or two briefly presented target items. On different blocks of trials, observers were instructed to give different attentional priority to reporting the color versus the shape of the target item(s). This procedure enabled us to use descriptive methods, such as the performance operating characteristic (POC; Norman \& Bobrow, 1975) or the attention operating characteristic (AOC; Kinchla, 1969, 1980; Sperling \& Melchner, 1978a, 1978b), in order to get a complete picture of joint performance across instructions. Furthermore, a trial-by-trial contingency analy-

This research was supported by NIDCD Grant 07787 to Ervin Hafter and NSF Grant SBR-9319103 to W. Prinzmetal. We would like to thank Diane Beck, Alan Cooke, Erik Gallun, Erik Jensen, and Ethan Newby for their critical comments on aspects of the paper. Reprint requests should be sent to A.-M. Bonnel, Centre de Recherche en Psychologie Cognitive CNRS-CNRC, 31 Chemin Joseph Aiguier, 13402 Marseille, Cedex 09, France (e-mail: ambonnel@socrates.berkeley.edu). sis allowed us to test the independence of color and shape processes (Garner \& Morton, 1969) and to infer the attention allocation strategy used by observers (but see Sperling, 1984). Finally, psychophysical models, such as those derived from signal detection theory $\left(d^{\prime}\right.$; Green \& Swets, 1966/1974), were applied to the data to specify the mechanisms that might underlie the processing of simultaneous inputs.

The issue of whether observers can divide attention between color and shape begins with the observation that these are separable perceptual dimensions (Garner, 1974; Shepard, 1964). Logically, at least, they are separable in that shape remains invariant under color transformations. There is also neurophysiological evidence for separate cortical maps tuned specifically to color and shape (Cowey, 1985). Computationally, quite different kinds of operations are needed in the analysis of different visual dimensions (e.g., Ullman, 1984). Functionally, separate maps for color and shape are postulated by many current theories of attention (e.g., Cave \& Wolfe, 1990; Treisman \& Gelade, 1980). Thus, from some perspectives, it may appear natural that these separable dimensions should be independent, and indeed there does exist considerable evidence that color and shape are, at some level of analysis, processed separately (see Prinzmetal \& MillisWright, 1984, for a review).

Although the analyses of color and shape are in some respects independent, the question remains as to whether or not attention to one attribute affects performance on the other attribute. Early structural models, based on independent analyzers, suggest that attention to one attribute would not influence performance on the other (Allport, 1971; Broadbent, 1958; Treisman, 1969). These authors suggest that such dimensions as color and shape 

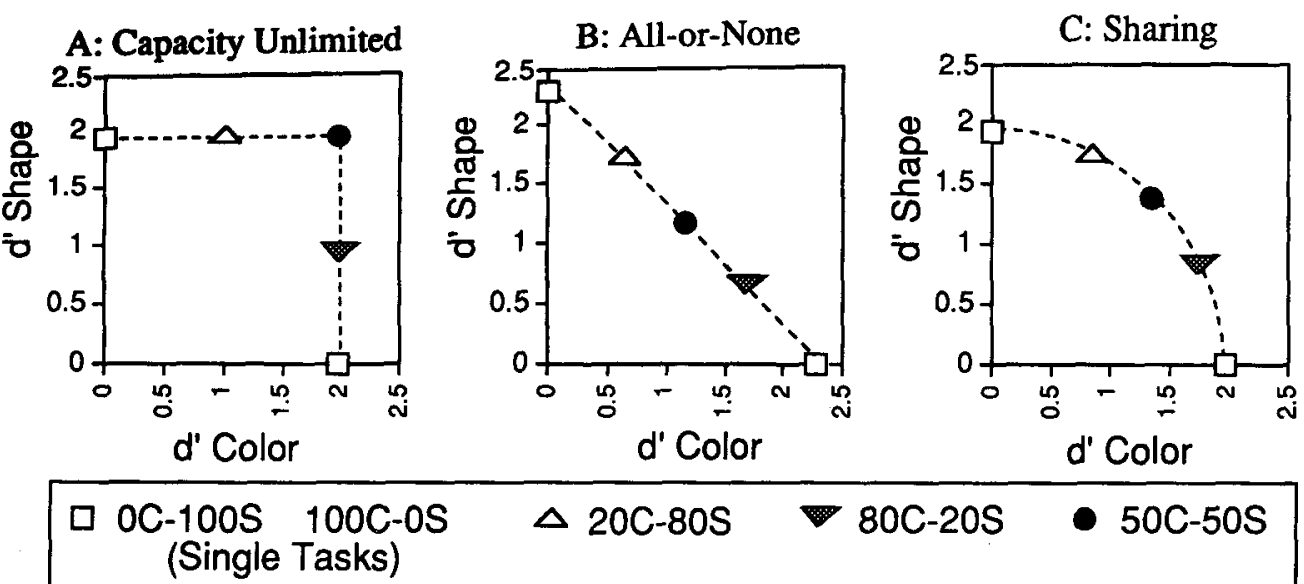

$\triangle 20 \mathrm{C}-80 \mathrm{~S}$

80C-20S

$50 \mathrm{C}-50 \mathrm{~S}$

Figure 1. Theoretical attentional operating characteristics (AOCs) for three situations: (A) tasks that do not use the same resources; (B) tasks that use the same resources; and (C) tasks that share some of the same resources.

might be processed by separate perceptual analyzers. Interference arises only when the task involves competition within the same population of analyzers. Thus, a task that involves the identification of color and shape should not show interference between dimensions. Furthermore, instructions to preferentially attend to one dimension should not affect performance on the other dimension. Data showing statistical independence between color and shape have been reported in cuing experiments (Isenberg, Nissen, \& Marchak, 1990; Nissen, 1985), although these data have been recently questioned (Monheit \& Johnston, 1994). Comparing single- and dual-task conditions, Allport (1971) showed that performance on a single task (e.g., the report of a color) was nearly as good as it was when paired with the report of a shape. Thus, the separate dimensions did not appear to conflict.

Recently, Duncan and Nimmo-Smith (1996) have found additional evidence supporting the analyzer hypothesis. They compared single- and dual-task performance for color and shape (line length), and found that the identification of the color of one object was not affected by a concurrent identification of the shape of another object. However, the identification of the color of one object was detrimentally affected by the concurrent identification of the color of the other object. Thus, a shape judgment did not interfere with a color judgment, but two color judgments did cause a dual-task decrement. Note, however, that they did not find this same dissociation between shape and brightness or texture.

In contrast to the early structural models, two classes of models predict that although color and shape are processed separately, attention to one attribute may, under certain circumstances, influence performance on the other attribute. These are the space-based and objectbased models of attention. Space-based models such as mental spotlight or zoom-lens theories assume that focal attention is assigned to restricted and continuous regions of the visual field (Broadbent, 1982; Eriksen \& Hoffman, 1973; Hoffman \& Nelson, 1981; Posner, Snyder, \& David- son, 1980). At a given moment, attention is focused on a particular area of visual space, and only stimuli within this area receive full perceptual analysis. Spotlight or zoom-lens theories predict that attention cannot be focused on noncontiguous locations at the same time. Thus, if the color and shape to be discriminated are spatially contiguous (or are part of the same object), attention to one attribute will not affect performance on the other attribute. However, if the color to be discriminated is in one location and the shape in another, it will be difficult to attend to both at the same time.

Object-based models assume that attention is assigned to an object or to perceptual groups rather than to continuous regions of the visual field (see, e.g., Banks \& Prinzmetal, 1976; Duncan, 1984, 1993; Egly, Driver, \& Rafal, 1994; Kahneman, 1973; Prinzmetal, 1981; Prinzmetal \& Banks, 1977). According to these models, an object's different attributes may be processed by separate subsystems, but selective attention is then a coordinate state in which the outputs of multiple subsystems are made available for the control of behavior. Moreover, an object's different attributes are always in the same attentional state, because focused attention on a particular object results in the mandatory processing of all attributes of that object. Thus, all attributes of an object are processed without interference. However, attention can be allocated separately to different objects.

The space-based and object-based models share the idea that whether or not attention to one attribute (e.g., color) affects performance on another attribute (e.g., shape) depends on the particular display. On the one hand, if the attributes are part of the same object or are in the same region of space, attention to one attribute will not affect performance on the other. On the other hand, if the attributes are from different objects or spatial regions, attention to one attribute will adversely affect performance on the other. In the present study, the predictions of the two classes of models would be the same, since objects and space covary and no particular attempt is made 

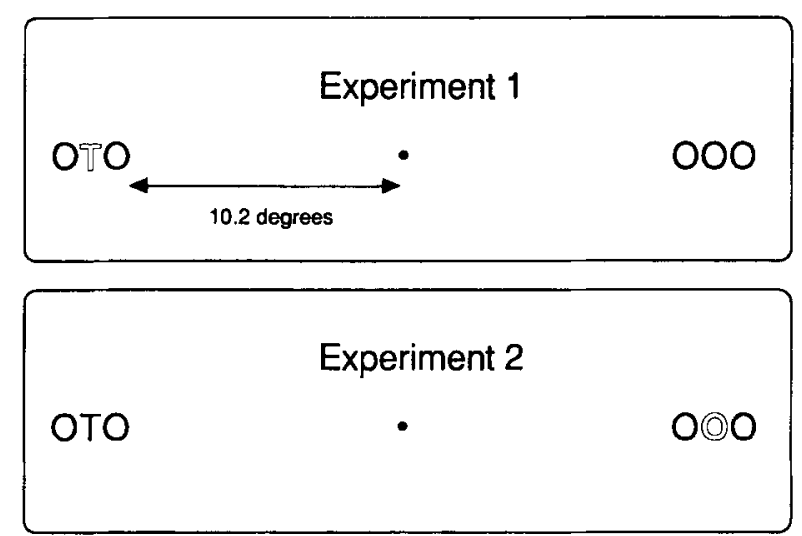

Figure 2. Sample stimuli from Experiments 1 and 2. The outlined letter was colored either green or blue.

to distinguish the number of objects from the number of locations. Both space-based and object-based theories can be contrasted with structural theories that propose that, to the extent that attributes are analyzed by independent analyzers, performance on one attribute will not influence performance on others, regardless of the spatial layout of the stimulus array.

There is evidence consistent with the space- and objectbased models. It has been shown, for example, that two identifications can be made without loss of accuracy provided the identification involves attributes of the same object (Duncan, 1984; Kahneman \& Henik, 1977; Treisman, Kahneman, \& Burkell, 1983). Similarly, the identification of two stimuli has been shown to be best when the stimuli are close together (Hoffman, Houck, Macmillan, Simons, \& Oatman, 1985; Hoffman \& Nelson, 1981; Kramer, Wickens, \& Donchin, 1985). These data suggest that efficient processing can occur on perceptual units that are organized either by preattentive processes or over a narrow area of space.

In the present experiments, observers were asked to identify the color and shape of target items. We compared performance in five attentional conditions in which observers were to devote $100 \%, 80 \%, 50 \%, 20 \%$, or $0 \%$ of their attention to identifying the target color and, correspondingly, $0 \%, 20 \%, 50 \%, 80 \%$, or $100 \%$ of their attention to identifying the target shape.

Several possible outcomes can be distinguished, as schematically shown in Figure 1. According to the early structural models, there should be no capacity limits on performance in color and shape identification, because color and shape require two separate pools of processing resources. This assumption predicts that performance will fall along the dashed line in the attention operating characteristic (AOC) depicted in Figure $1 \mathrm{~A}$. In this AOC curve, performance on one task is not affected by the second task, and so dual-task performance does not fall below that of the single-task condition. Furthermore, in a trial-by-trial analysis, accuracy on one task should not affect performance on the other. These predictions hold when the two attributes are parts of the same object and when they are parts of different objects.

At the other extreme, attention to both attributes at the same time might be impossible. Under this "all-or-none" strategy, observers can identify only one attribute on a given trial. On some trials, observers might attend only to the color, and on other trials, only to the shape. Given the instructions to devote $80 \%$ of their attention to the color and $20 \%$ to the shape, observers might attend exclusively to the color on $80 \%$ of the trials, yielding the performance function illustrated in Figure 1B. In a trialby-trial analysis, accuracy on one task should be negatively correlated with performance on the other (Sperling \& Melchner, 1978a, 1978b).

Finally, there are situations in which attention can truly be shared, resources being allocated differentially to the competing sources of information. The AOC for shared attention is illustrated in Figure 1C. If observers are sharing attention between color and shape identification within trials, a trial-by-trial analysis should not yield a correlation between tasks.

Most visual experiments using the concurrent-task paradigms have examined the effects of dividing attention between spatially distinct objects. These studies typically find a marked dual-task deficit, indicating that both tasks compete for resources. The attention strategy found in these studies corresponds predominantly to a simultaneous division of resources between concurrent tasks, as illustrated in Figure 1C (Baron, 1973; Bonnel \& Miller, 1994; Bonnel, Possamaï, \& Schmitt, 1987; Bonnel, Stein, \& Bertucci, 1992; Castiello \& Umiltà, 1990, 1992; Egly \& Homa, 1984; Hughes, 1984; Miller \& Bonnel, 1994; Müller \& Findlay, 1987; Pylyshyn \& Storm, 1988).

In summary, if the structural theories are correct, we should obtain results indicative of independence (Figure 1A) regardless of whether the color and shape are from the same object. If the object-based or space-based theories are correct, we should obtain evidence consistent with independence (Figure 1A) when the two attributes are from the same object. However, when the color is in one spatial location and the shape is in another, we should obtain results indicative of an all-or-none strategy (Figure 1B) or results indicating that observers are sharing resources between color and shape identification (Figure 1C). In Experiment 1, observers had to determine whether a single target letter was the letter $F$ or the letter $\mathrm{T}$. In addition, they had to determine whether this target letter was blue or green. In Experiment 2, observers had to determine whether a target letter was an $F$ or a $T$ and whether another letter (the letter $\mathrm{O}$ ) was blue or green. In Experiment 2, the letter and color targets were widely spaced; they were in different visual fields.

\section{EXPERIMENT 1 Same Object}

\section{Method}

Procedure. Each trial began with the presentation of a white fixation dot in the center of a computer monitor. After $0.5 \mathrm{sec}$, the 
Table 1

Accuracy From Experiments 1 and 2

\begin{tabular}{|c|c|c|c|c|c|c|c|c|}
\hline \multirow[b]{3}{*}{ Observer } & \multicolumn{4}{|c|}{ Experiment 1} & \multicolumn{4}{|c|}{ Experiment 2} \\
\hline & \multicolumn{2}{|c|}{ Color } & \multicolumn{2}{|c|}{ Shape } & \multicolumn{2}{|c|}{ Color } & \multicolumn{2}{|c|}{ Shape } \\
\hline & $\%$ Corr. & $d^{\prime}$ & $\%$ Corr. & $d^{\prime}$ & $\%$ Corr. & $d^{\prime}$ & $\%$ Corr. & $d^{\prime}$ \\
\hline \multicolumn{9}{|c|}{ Single Tasks } \\
\hline 1 & 82.3 & 1.82 & 83.5 & 1.94 & 83.7 & 1.97 & 87.8 & 2.34 \\
\hline 2 & 81.4 & 1.75 & 85.8 & 2.16 & 78.5 & 1.54 & 95.8 & 3.50 \\
\hline 3 & 78.5 & 1.58 & 80.7 & 1.75 & 75.3 & 1.21 & 77.4 & 1.48 \\
\hline 4 & 73.3 & 1.22 & 76.9 & 1.48 & 73.3 & 1.21 & 76.7 & 1.48 \\
\hline 5 & 78.1 & 1.54 & 80.0 & 1.68 & 79.9 & 1.68 & 76.7 & 1.48 \\
\hline 6 & 82.8 & 1.89 & 85.2 & 2.08 & 80.2 & 1.68 & 80.2 & 1.68 \\
\hline Mean & 79.4 & 1.63 & 82.0 & 1.85 & 78.5 & 1.55 & 82.4 & 1.99 \\
\hline \multicolumn{9}{|c|}{$20 \mathrm{C} / 80 \mathrm{~S}$ Condition } \\
\hline 1 & 82.8 & 1.89 & 83.5 & 1.94 & 64.6 & 0.76 & 86.8 & 2.26 \\
\hline 2 & 69.4 & 1.00 & 90.5 & 2.62 & 73.6 & 1.27 & 90.9 & 2.68 \\
\hline 3 & 78.3 & 1.54 & 85.4 & 2.08 & 61.1 & 0.56 & 77.8 & 1.54 \\
\hline 4 & 51.2 & 0.06 & 76.2 & 1.41 & 50.7 & 0.06 & 78.5 & 1.54 \\
\hline 5 & 79.5 & 1.65 & 82.3 & 1.82 & 72.2 & 1.16 & 84.0 & 1.97 \\
\hline 6 & 84.4 & 1.98 & 83.3 & 1.89 & 72.2 & 1.16 & 80.9 & 1.75 \\
\hline Mean & 74.3 & 1.35 & 83.5 & 1.96 & 65.7 & 0.83 & 83.2 & 1.96 \\
\hline \multicolumn{9}{|c|}{$50 \mathrm{C} / 50 \mathrm{~S}$ Condition } \\
\hline 1 & 78.3 & 1.54 & 83.2 & 1.89 & 69.1 & 1.00 & 79.9 & 1.68 \\
\hline 2 & 80.7 & 1.75 & 83.5 & 1.94 & 87.5 & 2.26 & 90.6 & 2.68 \\
\hline 3 & 79.5 & 1.65 & 83.7 & 1.98 & 70.5 & 1.04 & 70.5 & 1.04 \\
\hline 4 & 67.9 & 0.93 & 77.4 & 1.48 & 58.7 & 0.45 & 62.5 & 0.61 \\
\hline 5 & 79.5 & 1.65 & 78.6 & 1.61 & 71.9 & 1.16 & 77.1 & 1.48 \\
\hline 6 & 79.7 & 1.68 & 82.8 & 1.89 & 67.7 & 0.93 & 75.3 & 1.34 \\
\hline Mean & 77.6 & 1.53 & 81.5 & 1.80 & 70.8 & 1.14 & 76.0 & 1.47 \\
\hline \multicolumn{9}{|c|}{$80 \mathrm{C} / 20 \mathrm{~S}$ Condition } \\
\hline 1 & 84.7 & 2.08 & 82.3 & 1.82 & 77.0 & 1.48 & 71.0 & 1.10 \\
\hline 2 & 82.3 & 1.82 & 74.1 & 1.27 & 81.6 & 1.82 & 81.6 & 1.82 \\
\hline 3 & 86.8 & 2.26 & 76.9 & 1.48 & 76.4 & 1.41 & 54.9 & 0.25 \\
\hline 4 & 71.9 & 1.16 & 54.3 & 0.20 & 64.9 & 0.76 & 49.7 & 0.00 \\
\hline 5 & 78.5 & 1.58 & 79.9 & 1.68 & 76.7 & 1.48 & 80.9 & 1.75 \\
\hline 6 & 81.4 & 1.75 & 81.9 & 1.82 & 69.4 & 1.00 & 79.9 & 1.68 \\
\hline Mean & 80.9 & 1.77 & 74.9 & 1.38 & 74.3 & 1.32 & 69.6 & 1.10 \\
\hline
\end{tabular}

fixation dot was briefly replaced by two strings of three letters, one string on each side of the center of the monitor (see Figure 2). The letters remained in view for $83.3 \mathrm{msec}$ ( 5 computer refresh cycles at $60 \mathrm{~Hz}$ ) and the fixation dot reappeared. The center letter of one of the strings was the target, which could be either the letter $F$ or the letter $T$ and was green or blue. Each of the four possible targets appeared an equal number of times within a block, and the order of trials was determined randomly. The remaining letters were all the letter $\mathrm{O}$ and were white. The location of the target-letter string (left or right of fixation) was determined randomly.

There were five attention conditions; two were single tasks and three were dual tasks. In the $0 \mathrm{C} / 100 \mathrm{~S}$ (shape only) condition, observers were to respond only as to whether the letter target was the letter $\mathrm{F}$ or the letter $\mathrm{T}$. Thus, in this condition, observers were to devote $100 \%$ of their attention to determining the target shape $(\mathrm{F}$ or T). Similarly, in the $100 \mathrm{C} / 0 \mathrm{~S}$ condition (color only), observers were to respond as to whether the color target was blue or green. In the $20 \mathrm{C} / 80 \mathrm{~S}$ condition, observers were instructed to devote $20 \%$ of their effort to determining the color of the target and $80 \%$ to the shape of the target. In the $50 \mathrm{C} / 50 \mathrm{~S}$ condition, observers were to devote equal attention to determining the color and shape of the target. Finally, in the $80 \mathrm{C} / 20 \mathrm{~S}$ condition, the observers were to devote $80 \%$ of their attention to identifying the target color and only $20 \%$ to identifying the target shape.

Observers indicated their responses by pressing one of four buttons on a response panel. The buttons were situated in a diamond configuration and were labeled "Green F," "Green T," "Blue F," and "Blue T." In conditions in which both the color and shape of the target were to be identified, the observers responded by pressing a single button which indicated both the color and shape identity of the target. In the $100 \mathrm{C} / 0 \mathrm{~S}$ condition (color only), the observers could indicate the color of the target by pressing either the button labeled "green" or the button labeled "blue." Similarly, in the $100 \mathrm{C} /$ OS condition (shape only), the observers indicated the identity of the target shape in a similar manner. The observers were instructed to maximize their accuracy; reaction time was not measured.

After making a response, the observers were given immediate feedback. In the conditions in which the observers were to indicate both color and shape, their responses were followed by two tones. The first tone indicated accuracy for the target color and the second tone indicated accuracy for the target shape. A highpitched tone indicated a correct response, a low-pitched tone indicated an incorrect response. Thus, two high-pitched tones indicated a correct response for both the color and shape. A high-pitched tone followed by a low-pitched tone indicated a correct color response and an incorrect letter-shape response, and so on. In conditions in which observers indicated only the target color or only the target shape, the response was followed by a single tone. At the end of a block of trials, the observers were informed of their accuracy in determining the color and/or letter for the whole block.

The five attention conditions were varied between blocks of trials. Before each block, the observers were given verbal in- 


\section{Observer 1}

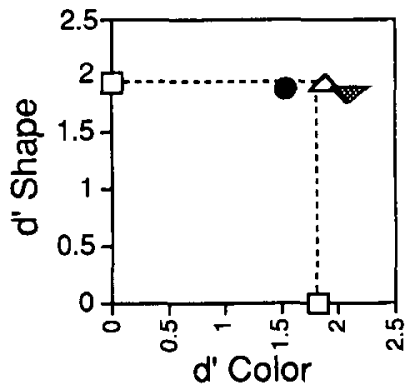

Observer 4

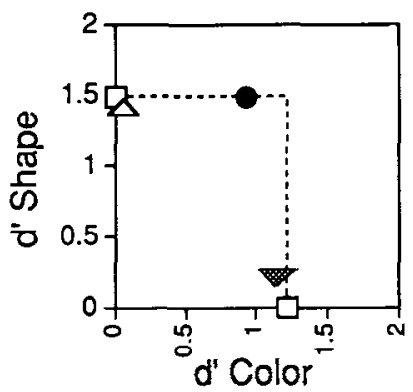

OC-100S 100C-0S (Single Tasks)
Observer 2

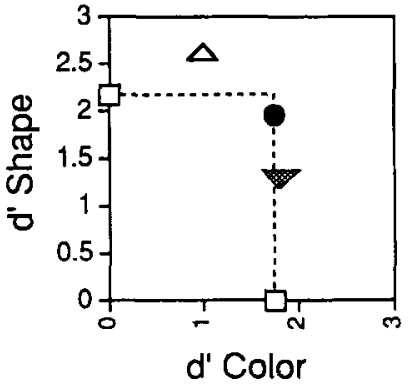

Observer 5

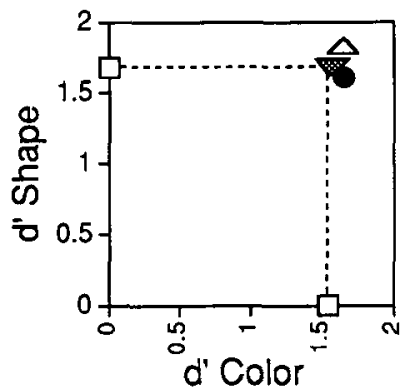

$\Delta 20 \mathrm{C}-80 \mathrm{~S}$
Observer 3

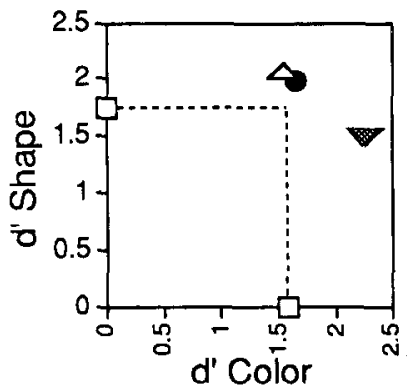

Observer 6

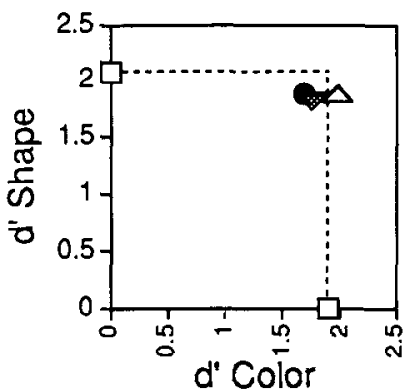

$80 \mathrm{C}-20 \mathrm{~S}$

50C-50S

Figure 3. AOC curves from Experiment 1. The squares denote single-task performance; the circles, the $50 \mathrm{C} / 50 \mathrm{~S}$ condition; the upward-pointing triangles, the $20 \mathrm{C} / 80 \mathrm{~S}$ condition; and the downward-pointing triangles, the $80 \mathrm{C} / 20 \mathrm{~S}$ condition. Each point represents 576 trials.

structions as to which dimension they were supposed to attend. These priority instructions were given by using a simple ratio. For example, in the $20 \mathrm{C} / 80 \mathrm{~S}$ condition, they were told to allocate $20 \%$ of their attention to identifying the target color (green or blue) and $80 \%$ to identifying the target shape ( $F$ or $\mathrm{T}$ ).

Each observer participated in 12 blocks for each condition. The order of the conditions was randomized both within and across observers. The observers ran approximately six blocks in each 1-h session. Thus, for each condition, each observer was tested on 576 trials in approximately $101-\mathrm{h}$ sessions. The observers received at least $1 \mathrm{~h}$ of practice before the first session on which data were collected.

Stimuli. The stimuli were presented on an Apple 13-in. color monitor controlled by a Macintosh computer. The monitor had a screen resolution of 72 pixels per inch (approximately 28 pixels per centimeter). The observers were restrained with a chinrest, and they viewed the monitor from $40 \mathrm{~cm}$. The room was illuminated by florescent lighting.

In each block of 48 trials, there were 12 trials with each of the following targets: (1) green T, (2) green $F,(3)$ blue T, and (4) blue F. The order of trials within a block and the location of the target item (i.e., left or right string) were determined randomly.

The letters were all 24-pt Helvetica. The distance from the center of the monitor to the inside edge of the letter string covered an area of approximately $1.7 \times 0.6 \mathrm{~cm}\left(2.42^{\circ} \times 0.86^{\circ}\right.$ of visual angle). The distance from the center of the monitor to the inside edge of the letter strings subtended a visual angle of $10.2^{\circ}$

Since our aim was for performance approximately midway between chance and perfect, the exposure durations were selected on the basis of extensive pilot work. First, we determined an ex- posure duration that would lead to approximately $75 \%$ correct responding in the shape-only condition. For each observer, this was $83 \mathrm{msec}(5 \mathrm{refresh}$ cycles at $60 \mathrm{~Hz}$ ). Second, we varied saturation of the green and blue stimuli until each observer's color-only performance (Condition $100 \mathrm{C} / 0 \mathrm{~S}$ ) approximately matched their shape-only performance (Condition $0 \mathrm{C} / 100 \mathrm{~S}$ ). After these parameters were established, data collection began and the exposure conditions remained constant throughout the experiment. For 4 of the observers, the CIE coordinates and luminance values of the green and blue stimuli were $x=.300, y=.359,39.5 \mathrm{~cd} / \mathrm{m}^{2}$ and $x=$ $.260, y=.243,29.8 \mathrm{~cd} / \mathrm{m}^{2}$, respectively. For 1 observer, the CIE coordinates and luminance values of the green and blue stimuli were $x=.304, y=.403,36.6 \mathrm{~cd} / \mathrm{m}^{2}$ and $x=.240, y=.210,22.0 \mathrm{~cd} / \mathrm{m}^{2}$, respectively. These measurements were made by filling the screen with the color and using a Minolta CS photometer. These shades of green and blue are very similar and difficult for an unpracticed observer to discriminate. The luminance of the white letters was $75.0 \mathrm{~cd} / \mathrm{m}^{2}$ (when measured over a solid area), and that of the black background of the monitor was $6.5 \mathrm{~cd} / \mathrm{m}^{2}$.

Observers. Six observers participated in this experiment $(2 \mathrm{fe}$ male). All the observers were undergraduates at the University of California, Berkeley, and their ages ranged from 18 to 24 years. All were well practiced in psychophysical experiments, but there were no other selection criteria. They were paid by the hour for their participation.

\section{Results and Discussion}

For the data analysis, the results were collapsed over the target color (green or blue) and target shape ( $\mathrm{F}$ or $\mathrm{T})$. 


\begin{tabular}{|c|c|c|c|c|c|c|}
\hline Observer & Color & Shape & Both & Indep & Gamma & $x^{2}$ \\
\hline \multicolumn{7}{|c|}{$20 \mathrm{C} / 80 \mathrm{~S}$ Condition } \\
\hline 1 & .828 & .835 & .733 & .691 & .677 & $264,40^{*}$ \\
\hline 2 & .694 & .905 & .639 & .628 & .267 & $41.06^{*}$ \\
\hline 3 & .783 & .854 & .679 & .669 & .215 & $26.68^{*}$ \\
\hline 4 & .512 & .762 & .399 & .390 & .100 & $5.76 \dagger$ \\
\hline 5 & .795 & .823 & .694 & .654 & .608 & $212.85^{*}$ \\
\hline 6 & .844 & .833 & .746 & .703 & .720 & $298.60^{*}$ \\
\hline Mean & .743 & .835 & .648 & .623 & .431 & $141.56^{*}$ \\
\hline \multicolumn{7}{|c|}{ 50C/50S Condition } \\
\hline 1 & .783 & .832 & .693 & .651 & 624 & $224.28 *$ \\
\hline 2 & .807 & .835 & .701 & .674 & .490 & $138.51^{*}$ \\
\hline 3 & .795 & .837 & .677 & .665 & .237 & $32.32 *$ \\
\hline 4 & .679 & .774 & .564 & .525 & .445 & $114.14^{*}$ \\
\hline 5 & .795 & .786 & .679 & .625 & .680 & $267.19^{*}$ \\
\hline 6 & .797 & .828 & .715 & .660 & .750 & $324.34^{*}$ \\
\hline Mean & .776 & .815 & .671 & .633 & .538 & $183.46^{*}$ \\
\hline \multicolumn{7}{|c|}{$80 \mathrm{C} / 20 \mathrm{~S}$ Condition } \\
\hline 1 & .847 & .823 & .743 & .697 & .740 & $310.15^{*}$ \\
\hline 2 & .823 & .741 & .625 & .609 & .245 & $34.62^{*}$ \\
\hline 3 & .868 & .769 & .677 & .667 & .212 & $25.93^{*}$ \\
\hline 4 & .719 & .543 & .380 & .390 & -.100 & $5.76 \div$ \\
\hline 5 & .785 & .799 & .684 & .627 & .713 & $292.86^{*}$ \\
\hline 6 & .814 & .819 & .717 & .667 & .714 & $293.87^{*}$ \\
\hline Mean & .809 & .749 & .638 & .609 & .421 & $160.53^{*}$ \\
\hline
\end{tabular}

The proportion correct and $d^{\prime}$ (from signal detection theory) for each observer and attention condition is shown in Table 1. Assuming a symmetrical criterion (no preference for F vs. T or green vs. blue), then $d^{\prime}=2 * z$ [p(correct)].

First, we note that the single-control tasks are very similar to each other, although there is a slight superiority of shape identification $\left(d^{\prime}=1.85\right)$ over color $\left(d^{\prime}=\right.$ 1.63). As shown in Table 1, this difference, although true for each observer, is very small.

Next, we examined the shape of the AOC functions for each observer to determine whether observers were trading performance between the two tasks. The AOC functions are shown in Figure 3. If we examine the shape of the individual AOC curves observed in this experiment, a somewhat homogeneous picture emerges. There is no accuracy tradeoff between attentional conditions, indicative of a total absence of competition for resources between tasks (see Figure 1A). For every observer, simultaneous identification of color and shape is not impaired in comparison with the identification of color or shape alone.

However, a closer look at each function indicates that there are really two patterns of results. Three observers (Observers 1, 5, and 6) do not show any effect of the priority instructions. Their data fall around the so-called "independence point" located at the intersection of the two lines connecting single-task performance. ${ }^{1}$ While these observers do not exhibit attentional control, the same is not true for the other 3 observers $(2,3$, and 4$)$, whose performances are clearly ordered in agreement with the priority instructions. Moreover, Observer 4 exhibits a square $A O C$ indicative of independence, as does Observer 2. In contrast, Observer 3 shows a slight concur- rence benefit for each attentional instruction. Despite these different patterns of results, for each observer, performance on the dual-task conditions is as good as or better than single-task performance.

The $A O C$ is a useful tool for describing the joint performance on the two tasks over the experiment. However, it does not describe the relation between the tasks within a trial. Sperling (1984) suggested examining the correlation between performance on the two tasks on a trialby-trial basis in order to understand the processing mechanisms by which the observer moves along the AOC curve, that is, how attention is shifted from one dimension to the other (also see Bonnel \& Miller, 1994; Miller \& Bonnel, 1994; Sperling \& Melchner, 1978a, 1978b).

There are three possible outcomes of a trial-by-trial correlational analysis. First there might be a negative correlation between performance on the two tasks. A negative correlation might be taken to indicate that observers direct their attention toward one dimension on any given trial and alternate from dimension to dimension between trials. Trial-by-trial switching models predict a negative contingency between the accuracy of the two responses made on the same trial. When the response on one dimension is correct, the response on the other dimension is likely to be wrong, and vice versa.

A second possibility is that there would be little or no correlation between performance in the two tasks. This would indicate that observers were truly sharing resources within a trial between the two tasks. As pointed out in the introduction, the early structural models predict no correlation between performance on two structurally independent dimensions.

Finally, there might be a positive correlation between performance on the two tasks. This outcome would indicate that the two dimensions were somehow unified; that is, on a trial, if one perceived the shape, one would likely also perceive the color. Such an outcome would be consistent with the notion that color and shape (of one object) were in some ways integral dimensions.

For each priority instruction, the proportion of correct responses was calculated for color and shape for each observer. Table 2 presents color- and shape-component performance for each observer along with the observed probability of getting both correct. The adjacent column shows the independence prediction, $P_{\mathrm{AB}}=\left(P_{\mathrm{A}}\right)\left(P_{\mathrm{B}}\right)$ for each observer. For all but 1 observer, the observed conjunction performance exceeded the prediction from independence in each priority instruction (for 1 observer and one priority instruction only, i.e., $80 \mathrm{C} / 20 \mathrm{~S}$, conjunction performance is slightly lower than the prediction from independence). Next to this, another measure of dependence in a $2 \times 2$ contingency table is the gamma coefficient, or Yule's Q (Hays, 1981; Hintzman, 1992). Gamma is a correlation coefficient that ranges from -1 (total negative dependence) to +1 (total positive dependence). This measure is sensitive to unequal performance on two components. Of the 18 tests ( 6 observers $\times 3$ priority instructions), 17 were positive. Table 2 also shows the results of the chi-square tests. Of the 18 

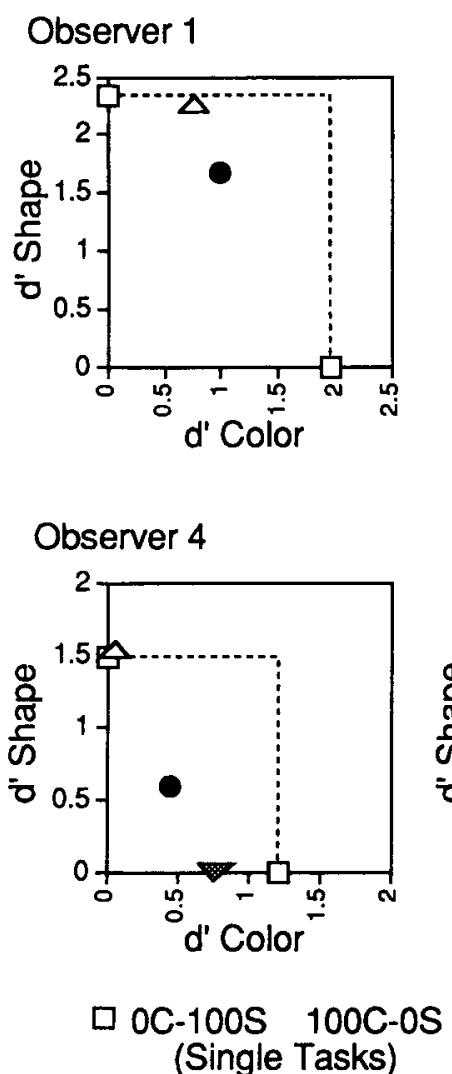
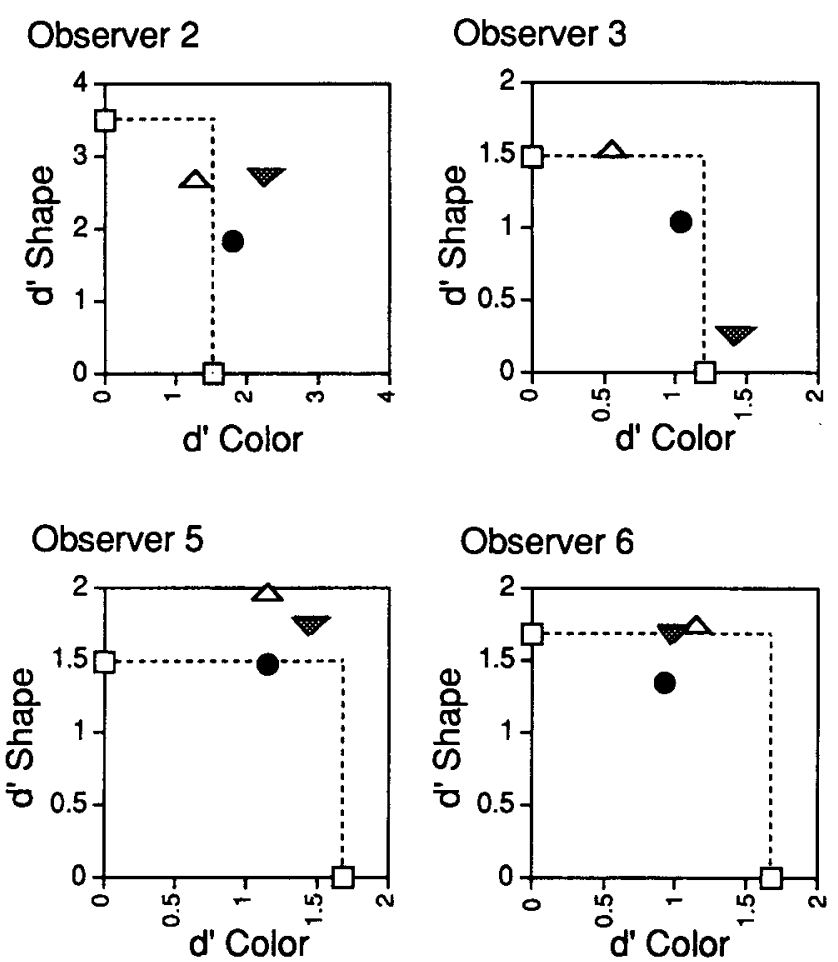

$\Delta$ 20C-80S

80C-20S
$50 \mathrm{C}-50 \mathrm{~S}$

Figure 4. AOC curves from Experiment 2. The squares denote single-task performance; the circles, the $50 \mathrm{C} / 50 \mathrm{~S}$ condition; the upward-pointing triangles, the $20 \mathrm{C} / 80 \mathrm{~S}$ condition; and the downwardpointing triangles, the $80 \mathrm{C} / 20 \mathrm{~S}$ condition. Each point represents 288 trials.

tests, 17 showed a significant departure from independence, 16 of which were significant $(p<.001)$.

Thus, we consistently obtained positive correlations between performance on the two tasks. Observers were more likely to be correct on one dimension if they were correct on the other. Interestingly enough, Table 2 also reveals that the positive dependency (gamma coefficient) was noticeably higher for Observers 1,5 , and 6, who performed with the same accuracy on both tasks in spite of priority instructions. In this group, the average gamma value is $.67, .68$, and .72 for each priority instruction $(20 \mathrm{C} / 80 \mathrm{~S}, 50 \mathrm{C} / 50 \mathrm{~S}$, and $80 \mathrm{C} / 20 \mathrm{~S}$, respectively). For the 3 other observers $(2,3$, and 4$)$, who were successful in imbalancing their attention between both dimensions, these values, although significantly positive, were only $.19, .39$, and .12. The lower dependence we observed in the second group of observers may have been due to an increase in the number of guesses about the low-priority dimension (see Monheit \& Johnston, 1994), and, indeed, their gamma coefficient is lower in these conditions than in the $50 \mathrm{C} / 50 \mathrm{~S}$ condition. Observer 4 , who exhibits a square AOC curve compatible with independent processing of both dimensions, does indeed show a negative contingency in the $80 \mathrm{C} / 20 \mathrm{~S}$ condition. ${ }^{2}$ Differences in strategy may be responsible for these individual patterns.
The results of Experiment 1 can be briefly summarized as follows. The AOC analysis does not show that color and shape perception share the same processing capacity, in that attention to one does not reduce performance on the other. However, the correlation analysis demonstrates that, in a trial-by-trial analysis, performance on color and shape is strongly correlated. The latter finding is inconsistent with the structural view that predicts no correlation between the perception of color and shape. Before rejecting the structural view, we need to consider two alternative explanations of the positive correlation between the two tasks.

First, the positive correlation between tasks could be caused by trial-to-trial fluctuations that subtly influence performance on both tasks in the same way (e.g., alertness, practice level) or by the existence of occasional trials on which observers give wrong answers for reasons extraneous to their perceptual processing (e.g., blinks). Such general trial-by-trial factors would always operate to increase the correlation between the two tasks.

Second, it is well known that the chi-square statistic computed from $2 \times 2$ tables only approximately follows the chi-square distribution (e.g., Marascuilo, 1971), and simulations of a model in which independence held (Miller \& Bonnel, 1994) revealed that Type I errors are much móre 


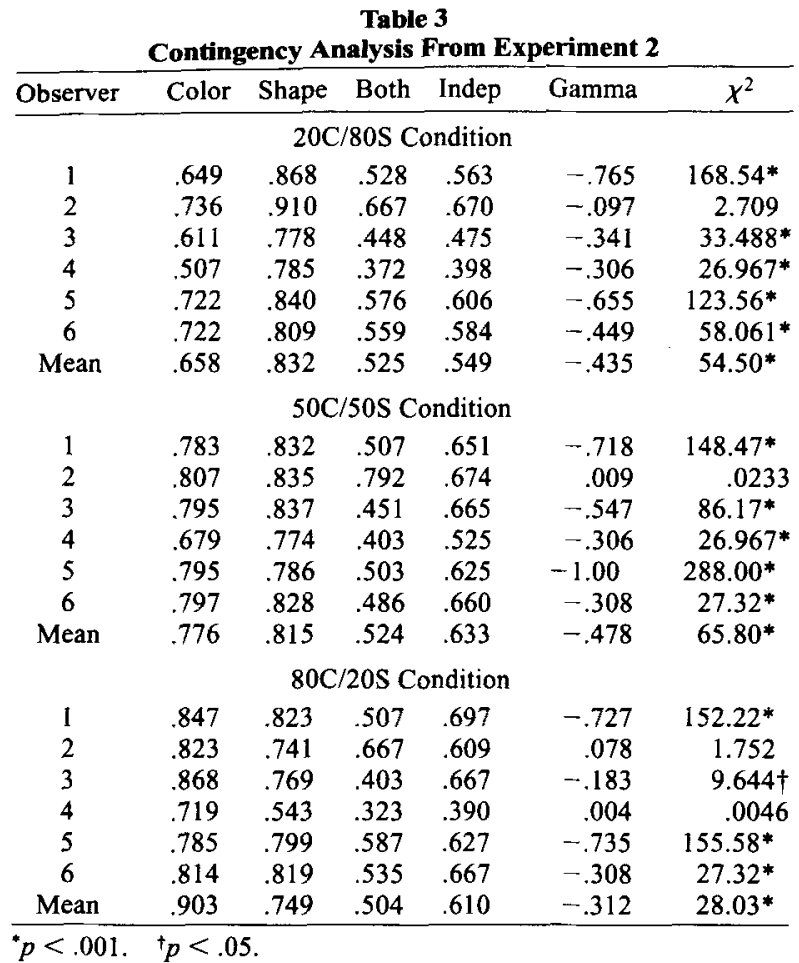

likely to occur with positive than with negative correlations, especially when the probability of a correct response exceeds .80 . Thus, positive correlations observed with a high percentage of correct responses could be due to Type I error probabilities.

In Experiment 2, we tested whether the above general factors caused the positive correlation between color and shape perception by creating a situation in which object and space-based theories do not predict a correlation between the two dimensions. Experiment 2 was identical to Experiment 1 except that the shape target ( $\mathrm{F}$ or $\mathrm{T}$ ) was monochromatic and in a location different from that of the color target (which was the letter $\mathrm{O}$ ). The general factors that might cause a positive correlation between color and shape perception should still operate. However, space and object-based theories predict that it should be difficult to simultaneously attend to two different objects in two different locations.

\section{EXPERIMENT 2 Different Objects}

\section{Method}

The methods were the same as those in the first experiment except that the target letter ( $F$ or $T$ ) was the center letter of one string of three letters and the color was the color of the center $O$ in the other string (see Figure 2). The location of the color and the letter was randomized, but the target color and the target letter were always in opposite visual fields. The 6 observers from Experiment 1 participated in Experiment 2 several weeks after completing Experiment 1 . The only difference in procedure was that to achieve approximately $75 \%$ correct, an exposure duration of only $51 \mathrm{msec}$ ( 3 refresh cycles) was necessary. The reason that we had to use a shorter exposure duration to achieve the same level of performance for Experiment 2, as compared with Experiment 1, may have been due to the fact that the observers were more practiced before beginning Experiment 2 than they had been before Experiment 1 .

\section{Results and Discussion}

The absolute level of single-task performance was very similar to that of Experiment 1 (see Table 1). Note, however, that the exposure duration was shorter in Experiment 2 ( 3 vs. 5 refresh cycles at $60 \mathrm{~Hz}$ ). As in Experiment 1 , observers were more accurate at the shape judgments than at the color judgments, but the difference was small.

The individual AOCs are presented in Figure 4. In general, the AOC functions show a cost of concurrence. For 5 of the 6 observers, the $50 \mathrm{C} / 50 \mathrm{~S}$ condition was worse than the single control. The only exception is an observer whose performance was particularly accurate (Observer 3$)$. However, the performance of 3 observers $(2,5$, and 6) did not vary with instruction, whereas the performance of three other observers $(1,3$, and 4$)$ clearly followed the priority instructions. The linear tradeoff exhibited by the latter observers is consistent with a strategy of resource allocation in which the totality of resources is allocated to one dimension at a time and switched across trials. For example, the $20 \mathrm{C} / 80 \mathrm{~S}$ instruction leads to the observer's devoting all of his or her attention to color on $20 \%$ of the trials. This pattern of results is most clearly seen in Observer 4 whose AOC intersects the single-task conditions (i.e., Figure 1B), thus showing a total incompatibility between the two tasks. Presumably, movement along the AOC is accomplished by switching between the two extreme attentional states. When $100 \%$ of attention is allocated to the color, very little knowledge is extracted about the shape of the letter. We will return to this issue of how much information is extracted from the unattended dimension after we present the trial-by-trial correlational analysis.

Note that our findings contradict those of Duncan and Nimmo-Smith (1996), who found no dual-task deficit when observers judged the color of one object and the shape of another. The discrepant findings may be due to differences in experimental details. For example, they termed as "shape" judgments what were really linelength judgments, while our shape judgments were letter identifications. Alternatively, we were more successful in equating single-task performance, while their observers were much more accurate at making color judgments than they were at making shape judgments. A ceiling effect on color performance could give the impression of independence. In any case, our results demonstrate that there can be a dual-task deficit when the shape of one object and the color of another are being judged.

We analyzed the trial-by-trial correlation between performance on the two tasks. These results, shown in Table 3 , are in marked contrast to those of Experiment 1. In Experiment 1, there was a positive correlation between performance on the two tasks: when observers were correct on the shape, they were also generally correct on the color. 
Table 4

Predicted and Observed Information From the Unattended Task

\begin{tabular}{|c|c|c|c|c|c|c|}
\hline \multirow[b]{3}{*}{ Observer } & \multicolumn{3}{|c|}{ Color } & \multicolumn{3}{|c|}{ Shape } \\
\hline & \multirow[b]{2}{*}{$100 \%$} & \multicolumn{2}{|c|}{$50 \%$} & \multirow[b]{2}{*}{$100 \%$} & \multicolumn{2}{|c|}{$50 \%$} \\
\hline & & Predicted & Observed & & Predicted & Observed \\
\hline 1 & 83.7 & 66.9 & 69.1 & 87.8 & 68.9 & 79.9 \\
\hline 2 & 78.5 & 64.2 & 87.5 & 95.8 & 72.9 & 90.6 \\
\hline 3 & 75.3 & 62.6 & 70.5 & 77.4 & 63.7 & 70.5 \\
\hline 4 & 73.3 & 61.6 & 58.7 & 76.7 & 63.3 & 62.5 \\
\hline 5 & 79.9 & 64.9 & 71.9 & 76.7 & 63.3 & 77.1 \\
\hline 6 & 80.2 & 65.1 & 67.7 & 80.2 & 65.1 & 75.3 \\
\hline
\end{tabular}

In Experiment 2, the results were the opposite. When observers were correct on one dimension, they were generally incorrect on the other dimension. Thus, when the color is in one location and the shape is in the other, allocating resources to both locations in a trial becomes impossible. As shown in Table 3, negative correlations are highly significant for 5 of the 6 observers whatever the allocation instructions $(50 \mathrm{C} / 50 \mathrm{~S}, 20 \mathrm{C} / 80 \mathrm{~S}$, or $80 \mathrm{C} / 20 \mathrm{~S})$.

By using both the $\mathrm{AOC}$ analysis and the correlational analysis, we can precisely infer how much information is extracted from an unattended stimulus. Suppose that, on each trial, an observer attends to one task or the otherbut never to both - and obtains information only about the attended task. As a consequence, this observer is merely guessing about each unattended stimulus. Given this assumption, performance in the $50 \mathrm{C} / 50 \mathrm{~S}$ condition can be predicted with the following formula:

$$
\begin{aligned}
P(C)_{50 \%}= & p_{\text {attended }} \times P(C)_{100 \%} \\
& +\left(1-p_{\text {attended }}\right) \times 0.5 .
\end{aligned}
$$

The predicted and observed values are shown in Table 4. Observer 2, who has a positive correlation between tasks (see Table 3), obviously extracted some information from both stimuli. Performance in the dual task is higher than predicted not only under an all-or-none model, but also in the single task for color identification. We also note that single performance is higher than the maximum achievable $(75 \%)$ if the observer responds perfectly on each trial's attended task. These results hold for each dimension (color and shape).

In clear contrast to this, Observer 4 switches between concurrent dimensions, and does it in an all-or-none fashion. For each dimension (color and shape), the observed performance is lower than what would be predicted by attending to only one task or the other, indicating that there is no information extracted from the unattended stimulus.

The remaining observers also switch (as shown by their negative contingencies), but they do obtain some information from the unattended stimulus. For these observers, we can reject the hypothesis that there are only two attentional states. The switching actually occurs between states of shared attention, or what has been called a strategy mixture (see Sperling \& Melchner, 1978a, 1978b). Thus, the trial-by-trial correlational analysis and the AOC analysis provide a convergent picture of observers' performance.
In general, the larger the negative correlation between performance on the two tasks, the more likely it is that the AOC conforms to a trial-by-trial switching strategy.

Further evidence concerning the attention strategy can be obtained by plotting the performance resource function (PRF; Norman \& Bobrow, 1975), in which percent correct is reported as a function of each attention instruction. If the all-or-none switching model is correct, the equation above predicts that the points should lie on a straight line connecting $0 \%$ to $100 \%$ attention. In Figure 5 , the data averaged over the 3 observers who seem to switch their attention between trials is shown for each dimension separately. The color data in Figure 5 fit quite well with the all-or-none switching model (dashed line). Such results indicate that little information was gained from the unattended dimension and that probability matching was successful. However, it is interesting to note that the data observed for the shape dimension (solid line), although linear, as predicted from probability matching, are located above the predicted values, thus demonstrating a so-called "concurrence benefit." Identification of color and shape does indeed improve performance relative to identification of the shape in isolation. Thus, when these observers attend to the color, they may also obtain some information about shape.

Although this probability-matching strategy is well described on a theoretical level, it is relatively uncommon.

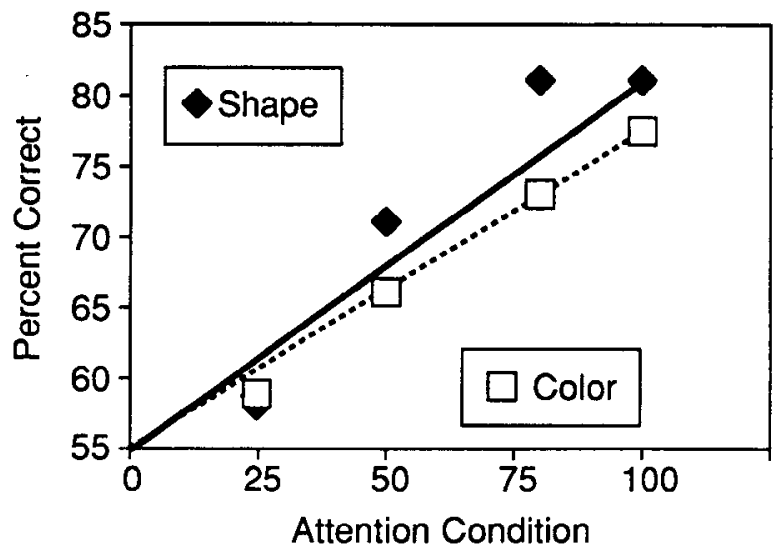

Figure 5. Performance resource function (PRF) for the 3 observers whose data most closely fit the all-or-none switching mode. 
Evidence of switching has been found only when observers must monitor multiple locations in order to detect a target letter (Shaw, 1982; Sperling \& Melchner, 1978a, $1978 \mathrm{~b}$ ). Evidence of sharing has been found when observers must monitor multiple locations to determine stimulus onset time (Baron, 1973) or brightness changes (Bonnel et al., 1992; Hughes, 1984; Pylyshyn \& Storm, 1988 ), or when they make line-length comparisons (Bonnel \& Miller, 1994; Miller \& Bonnel, 1994). This pattern of results suggests the intriguing hypothesis that attention is switched only when the task requires information about conjunctions of features (see Treisman, 1985). In the General Discussion, we will return to the issue of the conditions in which observers switch absolute attentional states (on a trial-by-trial basis) and when they share attention.

The results of Experiments 1 and 2, taken together, are clearly inconsistent with structural theories. In Experiment 1 , when the target color and form were part of the same object, there was a positive correlation between the two tasks and no cost of performing concurrent tasks. In Experiment 2, in which the target color and form were parts of different objects (in different locations), there was a negative correlation between the two tasks. The observers in Experiment 2 generally showed a cost of performing concurrent tasks (AOC analysis). Furthermore, the results from some of the observers in Experiment 2 could be fit to an all-or-none switching model, indicating that they could not perform the tasks concurrently.

Note that the difference between Experiments 1 and 2 is not due to the difficulty of each individual task. Experiment 2 was much easier than Experiment 1, as evidenced by the exposure duration used to meet the performance criteria. Nevertheless, Experiment 2 demonstrated more task interference. Furthermore, in Experiment 2, the observed dual-task deficit was not the result of monitoring two sources of information (Taylor, Lindsay, \& Forbes, 1967), since attention was not divided between the tasks. Rather, at least for some observers, attention was all or none. Consequently, the dual-task deficit must have been due to competition for processing resources, which is precisely denied by the structural theories.

\section{GENERAL DISCUSSION}

The results of the present research give strong support to object or space models and can be summarized as follows: Within an object, divided attention was easy and selective attention was impossible (i.e., Experiment 1). Furthermore, when observers perceived one attribute of an object (e.g., color), they were likely to perceive the other attribute (e.g., shape). When focal attention was paid to an object, all dimensions coded as properties of the whole were perceived without mutual interference. The results of Experiment 1 cannot be explained by general factors that might induce a positive trial-by-trial correlation in the perception of the two attributes. Experiment 2 was nearly identical to Experiment 1, but the attributes were parts of different objects in different locations. In
Experiment 2, however, there was a negative correlation in the perception of the two attributes. In summary, selective attention to one attribute was impossible in Experiment 1 , but selective attention to one attribute made it nearly impossible to attend to the other in Experiment 2.

Our design was similar to those of previous investigators (e.g., Duncan, 1984; Duncan \& Nimmo-Smith, 1996; Kramer, Weber, \& Watson, 1997; Vecera \& Farah, 1994), who reached similar conclusions. However, our method had several advantages over those of previous research. First, Duncan (1984), for example, compared only single- and dual-task performance. Single- and dual-task conditions differ in many respects other than the demands made on attention. By varying attention instructions, the AOC analysis describes an independent variable that is directly related to attention. Second, only by analyzing the AOC can we measure tradeoffs in the allocation of attention across tasks. Third, as shown by the present research, the AOC analysis by itself is inadequate to describe the processes that lead, or do not lead, to a performance tradeoff. The trial-by-trial correlation of performance across tasks reveals the attention strategy that underlies the AOC. As we have seen in Experiment 1, the AOC may not show a dual-task deficit, but that does not mean independence in the sense of correlational analysis. Reciprocally, in Experiment 2, the AOC may show a tradeoff, but that does not mean that attention was divided. We strongly recommend that investigators of dual-task performance examine these correlations.

Although the $\mathrm{AOC}$ and correlational analysis are logically orthogonal to each other, a somewhat homogeneous picture emerges from their use in the present experiments. On the one hand, within one object, identification of shape and color are perfectly compatible. On the other hand, between spatially separated objects, identification of shape and color are partially or totally incompatible, as shown by the performance impairment in the dual task. Finally, the AOC and correlational analysis could be used together to estimate the amount of information obtained for each attribute. The power of our methodology derived from (1) explicitly varying attention with instructions to generate the AOC, and (2) the use of trial-bytrial correlational analysis to help us understand the processes underlying the AOC.

There are two research areas that we would like to pursue. First, there are several possible differences between experiments that could have led to the positive correlation of feature identification in Experiment 1 and the negative correlation in Experiment 2. In Experiment 1, both the color and shape were in the same visual field, whereas in Experiment 2 the color and shape were in different visual fields. Hughes and Zimba (1985) claimed that attention was only to a visual field, but they did not have location specificity within a field. Since that report, several investigators have found that the distance between where observers are attending and the target within a visual field does affect performance (e.g., Downing \& Pinker, 1987; Henderson \& Macquistan, 1993; McCormick \& Klein, 1990). Of course, a second differ- 
ence between Experiments 1 and 2 was the distance between features: In Experiment 1, the features were in the same location; in Experiment 2, they were in different locations. Finally, in Experiment 1, the color and shape were part of the same object, whereas in Experiment 2 they were part of different objects. Several investigators have found that the distance between the stimuli in a dual-task experiment affects performance (e.g., Hoffman, Nelson, \& Houck, 1983; Kramer et al., 1997). Furthermore, performance is better when two attributes of the same object rather than attributes of different objects are being judged (e.g., Duncan, 1984, 1993; Lavie \& Driver, 1996). Unfortunately, these investigators have not looked at the correlations between tasks on a trial-bytrial basis.

The second question that we would like to pursue concerns when attention sharing is possible, yielding an $\mathrm{AOC}$ as in Figure 1C. A number of studies using the AOC methodology have demonstrated that attention can be divided either between spatial locations (Bonnel \& Miller, 1994; Bonnel et al., 1987; Bonnel et al., 1992; Miller \& Bonnel, 1994) or between sensory channels (Bonnel \& Hafter, in press). However, in Experiment 2, we might have expected that attention could be shared between the two attributes, as indicated in Figure $1 \mathrm{C}$ and as has been found by previous research. Instead, observers performed the tasks in an all-or-none fashion. We can think of at least two differences between Experiment 2 and the previous research. First, in most of the previous experiments on visual attention, observers always knew where to allocate attention before each trial, although attention instructions were also varied from trial to trial (Bonnel \& Miller, 1994). In the present experiments, the locations of the stimuli varied from trial to trial. In Experiment 2, for example, the observer in the $80 \mathrm{C} / 20 \mathrm{~S}$ condition must first locate the color before allocating attention in the proportions required. Perhaps there was not enough time to appropriately allocate attention once the color was found. Second, in previous experiments, attention was divided within rather than between dimensions. Kantowitz, Elvers, and Palmer (1991) obtained equivocal results in a paradigm in which attention was divided between line length and line orientation ( 2 observers shared attention and 2 observers switched attention in an all-or-none fashion). Third, the dimensions under study were different. Last, it is possible that, since color and shape are usually glued together in space, no common processing is possible when they are spatially separated.

In conclusion, in terms of attentional resources, the independence of attributes such as color and shape depended upon whether they were part of the same object (or in a similar location) or whether they were part of different objects (or in a different location). A complete picture of attention allocation was obtained only by varying attention between conditions, by considering the AOC, and by examining the trial-by-trial correlation between the perception of color and the perception of shape. The use of these techniques provides converging infor- mation on the processing strategies that underlie visual attribute processing.

\section{REFERENCES}

Allport, D. A. (1971). Parallel encoding within and between stimulus dimensions. Perception \& Psychophysics, 10, 104-108.

Banks, W. P., \& Prinzmetal, W. (1976). Configurational effects in visual information processing. Perception \& Psychophysics, 19, 361-367.

BARON, J. (1973). Perceptual dependence: Evidence for an internal threshold. Perception \& Psychophysics, 13, 527-533.

BONnel, A.-M., \& HAFTER, E. R. (in press). Divided attention between simultaneous auditory and visual signals. Perception \& Psychophysics.

BONNEL, A.-M., \& MiLLER, J. (1994). Attentional effects on concurrent psychophysical discriminations: Investigations of a sample-size model. Perception \& Psychophysics, 55, 162-179.

Bonnel, A.-M., Possamaï, C., \& Schmitt, M. (1987). Early modulation of visual input: A study of attentional strategies. Quarterly Journal of Experimental Psychology, 39A, 757-776.

Bonnel, A.-M., Stein, J. F., \& Bertucci, P. (1992). Does attention modulate the perception of luminance changes? Quarterly Journal of Experimental Psychology, 44A, 601-626.

Broadbent, D. E. (1958). Perception and communication. London: Pergamon.

Broadbent, D. E. (1982). Task combination and selective intake of information. Acta Psychologica, 50, 253-290.

Castiello, U., \& Umiltà, C. (1990). Size of the attentional focus and efficiency of processing. Acta Psychologica, 73, 195-209.

Castiello, U., \& Umiltà, C. (1992). Splitting focal attention. Journal of Experimental Psychology: Human Perception \& Performance, 18, 837-848.

Cave, K. R., \& Wolfe, J. M. (1990). Modeling the role of parallel processing in visual search. Cognitive Psychology, 22, 225-271.

CowEy, A. (1985). Aspects of cortical organization related to selective attention and selective impairments of visual perception. A tutorial review. In M. I. Posner \& O. S. M. Marin (Eds.), Attention and performance $X I$ (pp. 41-62). Hillsdale, NJ: Erlbaum.

Downing, C., \& Pinker, S. (1987). The spatial structure of attention. In M. Coltheart (Ed.), Attention and performance XII (pp. 171-187). Hillsdale, NJ: Erlbaum.

DUNCAN, J. (1984). Selective attention and the organization of visual information. Journal of Experimental Psychology: General, 113, 501-517.

DuNCAN, J. (1993). Similarity between concurrent visual discriminations: Dimensions and objects. Perception \& Psychophysics, 54, $425-430$,

Duncan, J., \& Nimmo-Smith, I. (1996). Objects and attributes in divided attention: Surface and boundary systems. Perception \& Psychophysics, 58, 1076-1084.

EGLY, R., DRIVER, J., \& RAFAL, R. D. (1994). Shifting visual attention between objects and locations: Evidence from normal and parietal lesion subjects. Journal of Experimental Psychology: General, 123, 161-177.

EGLY, R., \& HomA, D. (1984). Sensitization of the visual field. Journal of Experimental Psychology: Human Perception \& Performance, 10, 778-793.

ERIKSEN, C. W., \& HofFMAN, J. E. (1973). The extent of processing of noise elements during selective encoding from visual displays. Perception \& Psychophysics, 154, 155-160.

GARNER, W. R. (1974). The processing of information and structure. Hillsdale, NJ: Erlbaum.

Garner, W. R., \& MORTON, J. (1969). Perceptual independence: Definitions, models, and experimental paradigms. Psychological Bulletin, 72, 233-259.

GreEN, D. M., \& Swets, J. A. (1974). Signal detection and psychophysics. New York: Wiley. (Original work published 1966)

HaYs, W. L. (1981). Statistics (3rd ed.). London: Holt, Rinehart \& Winston.

Henderson, J. M., \& Macquistan, A. D. (1993). The spatial distribution of attention following an exogenous cue. Perception \& Psychophysics, 53, 221-230. 
HiNTZMAN, D. L. (1992). Mathematical constraints and the "TulvingWiseman Law." Psychological Review, 99, 536-542.

Hoffman, J. E., Houck, M. R., Macmillan, F. W., Simons, R. F., \& OAtMan, L. C. (1985). Event-related potentials elicited by automatic targets: A dual-task analysis. Journal of Experimental Psychology: Human Perception \& Performance, 11, 50-61.

Hoffman, J. E., \& Nelson, B. (1981). Spatial selectivity in visual search. Perception \& Psychophysics, 30, 283-290.

Hoffman, J. E., Nelson, B., \& Houck, M. R. (1983). The role of attentional resources in automatic detection. Cognitive Psychology, $15,379-410$.

HugHes, H. C. (1984). Effects of flash luminance and positional expectancies on visual response latency. Perception \& Psychophysics, 36, 177-184.

Hugres, H. C., \& Zimba, L. D. (1985). Spatial maps of directed visual attention. Journal of Experimental Psychology: Human Perception \& Performance, 11, 409-430.

IsenberG, L., Nissen, M. J., \& MarchaK, L. C. (1990). Attentional processing and the independence of color and orientation. Journal of Experimental Psychology: Human Perception \& Performance, 6 , 869-878.

Kahneman, D. (1973). Attention and effort. Englewood Cliffs, NJ: Prentice-Hall.

KAHNEMAN, D., \& HENIK, A. (1977). Effect of visual grouping on immediate recall and selective attention. In S. Dornic (Ed.), Attention and performance VII (pp. 181-211). Hillsdale, NJ: Erlbaum.

Kantowitz, B. H., Elvers, G. C., \& Palmer, J. (1991, November). Using attention operating characteristics to calibrate inferences about performance operating characteristics. Paper presented at the Annual Meeting of the Psychonomic Society, San Francisco.

KINCHLA, R. A. (1969). An attention operating characteristic in vision (Tech. Rep. No. 29). Hamilton, ON: McMaster University, Department of Psychology.

KINCHLA, R. A. (1980). The measurement of attention. In R. S Nickerson (Ed.), Attention \& performance VIII (pp. 213-218). Hillsdale, NJ: Erlbaum.

Kramer, A. F., Weber, T. A., \& Watson, S. E. (1997). Object-based attentional selection-Grouped-arrays or spatially invariant representations? Comment on Vecera and Farrah (1994). Journal of Experimental Psychology: General, 126, 3-13.

Kramer, A. F., Wickens, C. D., \& Donchin, E. (1985). Processing of stimulus properties: Evidence for dual-task integrality. Journal of Experimental Psychology: Human Perception \& Performance, 11, 393-408.

LAVIE, N., \& DRIVER, J. (1996). On the spatial extent of attention in object-based visual selection. Perception \& Psychophysics, 58, 1238 1251.

Marascuilo, L. A. (1971). Statistical methods for behavioral science research. New York: McGraw-Hill.

McCormick, P. A., \& KleIN, R. (1990). The spatial distribution of attention during covert visual orienting. Acta Psychologica, 75, 225242.

Miller, J., \& Bonnel, A. M. (1994). Switching or sharing in dualtask line-length discrimination? Perception \& Psychophysics, 56, 431-446.

Monhert, M. A., \& Johnston, J. C. (1994). Spatial attention to arrays of multidimensional objects. Journal of Experimental Psychology: Human Perception \& Performance, 20, 691-708.

Müller, H. J., \& FINDlay, J. M. (1987). Sensitivity and criterion effects in the spatial cuing of visual attention. Perception \& Psychophysics, 42, 383-399.

NAVON, D., \& Gopher, D. (1979). On the economy of the human processing system. Psychological Review, 86, 214-255.

NisSEN, M. J. (1985). Accessing features and objects: Is location special?
In M. I. Posner \& O. S. M. Marin (Eds.), Attention and performance $X I$ (pp. 205-219). Hillsdale, NJ: Erlbaum.

Norman, D. A., \& Bobrow, D. G. (1975). On data-limited and resource-limited processes. Cognitive Psychology, 7, 44-64.

Posner, M., Snyder, C. R. R., \& Davidson, B. J. (1980). Attention and the detection of signals. Journal of Experimental Psychology. General, 109, 160-174.

Prinzmetal, W. (1981). Principles of feature integration in visual perception. Perception \& Psychophysics, 30, 330-340.

Prinzmetal, W., \& Banks, W. P. (1977). Good continuation affects visual detection. Perception \& Psychophysics, 21, 389-395.

Prinzmetal, W., \& Millis-Wright, M. (1984). Cognitive and linguistic factors affect visual feature integration. Cognitive Psychology, 16, 305-340.

Pylyshyn, Z. W., \& Storm, R. W. (1988). Tracking multiple independent targets: Evidence for a parallel tracking mechanism. Spatial Vision, 3, 179-197.

SHAW, M. L. (1982). Attending to multiple sources of information: I. The integration of information in decision making. Cognitive Psychology, 14, 353-409.

ShEPARD, R. N. (1964). Attention and the metric structure of the stimulus space. Journal of Mathematical Psychology, 1, 54-87.

SPERLING, G. (1984). A unified theory of attention and signal detection. In R. Parasuraman \& D. R. Davies (Eds.), Varieties of attention (pp. 103-181). New York: Academic Press.

SPERling, G., \& Melchner, M. (1978a). The attention operating characteristic: Examples from visual search. Science, 202, 315-318.

SPERLING, G., \& MELCHNER, M. (1978b). Visual search, visual attention, and the attention operating characteristic. In J. Requin (Ed.), Attention and performance VII (pp. 675-686). Hillsdale, NJ: Erlbaum.

TAYLOR, M. M., LiNDSAY, P. H., \& FORBES, S. M. (1967). Quantification of shared capacity processing in auditory and visual discrimination. Acta Psychologica, 27, 223-229.

Treisman, A. M. (1969). Strategies and models of selective attention. Psychological Review, 76, 282-299.

Treisman, A. M. (1985). Preattentive processing in vision. Computer Vision, Graphics, \& Image Processing, 31, 156-177.

Treisman, A. M., \& Gelade, G. (1980). A feature-integration theory of attention. Cognitive Psychology, 12, 97-136.

Treisman, A. M., Kahneman, D., \& Burkell, J. (1983). Perceptual objects and the cost of filtering. Perception \& Psychophysics, 33, $527-532$.

Ullman, S. (1984). Visual routines. Cognition, 18, 97-159.

VeCERA, S. P., \& FARAH, M. J. (1994). Does visual attention select objects or locations? Journal of Experimental Psychology: General, $123,146-160$.

\section{NOTES}

1. Since the AOC is usually used as a purely descriptive device, this point is generally called the "independence" point. However, because there is a high correlation in performance between the two tasks in the trial-by-trial analysis, this term would be misleading in the present study.

2. According to resource theory as proposed by Navon and Gopher $(1979$, p. 238$)$, "If the type of resource the task demands are completely disjoint, then the tasks should be capable of being performed in parallel. In that case, resources released by degrading performance on one task are irrelevant for the performance of the other, so their performance is completely independent. Such a situation is represented by a square AOC."

(Manuscript received September 16, 1996; accepted for publication December 20, 1996.) 